\title{
Professionalitätsentwicklung von Kursleitenden
}

\author{
Stefanie Jütten · Anne Strauch
}

Online publiziert: 24. Juni 2015

(c) Die Autor(en) 2015. Dieser Artikel ist auf Springerlink.com mit Open Access verfügbar.

\section{Rezension zu:}

Claudia Schepers (2014). Wenn Kursleitende lernen. Orientierungssuche im Rahmen einer individuellen Professionalitätsentwicklung. Waxmann Verlag, Münster, 27,90€, 224 Seiten, ISBN 978-3-8309-3018-1

Dass die Professionalisierung in der Erwachsenen-/Weiterbildung und insbesondere die Professionalität von Lehrenden viel beforschte Themen sind, zeigt sich in der Vielfalt der Veröffentlichungen. Diese reichen von Strukturen und Situation der Beschäftigung, Fortbildungsmöglichkeiten, Kompetenzanforderungen bis hin zu Fortbildungsinteressen, -bedarfen und Teilnahmestrukturen. Die Erkenntnis, dass Lehrende in der Erwachsenen-/Weiterbildung oft keine (erwachsenen)pädagogische Ausbildung haben, sondern ganz unterschiedliche Bildungshintergründe, führt zu einer besonderen Beachtung von Fortbildungen für Lehrende in ihrer kompensatorischen Funktion.

In dieser Veröffentlichung, die als Dissertation an der Universität Hamburg eingereicht wurde, wird der Blick auf die einzelnen Lernprozesse der Kursleitenden selbst und mögliche Lernwiderstände gerichtet. Das leitende Erkenntnisinteresse der Dissertation ist es, Erklärungsansätze für mögliche Lernwiderstände von Kursleitenden aufzudecken und so Antwort auf die Frage zu geben, warum gerade diejenigen, die selber unterrichten und lehren, vielfach nicht an Fortbildungen teilnehmen, um sich für ihre Berufstätigkeit weiterzubilden. Da die berufsbegleitende Fortbildung gerade dieser Gruppe eine wichtige Funktion für die Sicherung der Veranstaltungsqualität in

S. Jütten $(\bowtie) \cdot$ Dr. A. Strauch

Deutsches Institut für Erwachsenenbildung - Leibniz-Zentrum für Lebenslanges Lernen e.V., Heinemannstr. 12-14, 53175 Bonn

E-Mail: juetten@die-bonn.de

Dr. A. Strauch

E-Mail: strauch@die-bonn.de 
der Erwachsenen-/Weiterbildung bietet, ist das Thema der Arbeit von großer Bedeutung vor dem Hintergrund weiterer Professionalisierungsbemühungen.

Im ersten Kapitel ordnet Claudia Schepers ihre Fragestellung in den Kontext der Professionsforschung ein, definiert Begriffe und stellt relevante professionstheoretische Ansätze dar. Im zweiten Kapitel erfolgt ein Überblick über berufsbegleitende Fortbildungen von Kursleitenden und eine Debatte darüber, welche Interessen oder Barrieren Kursleitende in Bezug auf Fortbildungen haben könnten. In Kapitel drei setzt sich Schepers mit einer ausgewählten Lerntheorie auseinander, nämlich der von Klaus Holzkamp, und bezieht Grundlagen der ,subjektwissenschaftlichen Lerntheorie" auf ihren Forschungsgegenstand. In Kapitel vier widmet sie sich spezifischen Lernwiderständen und -barrieren sowie Gründen einer Nicht-Teilnahme an Weiterbildungskursen bei Kursleitern.

Die empirische Basis der Forschungsarbeit bildet eine Mikroanalyse, die in Anlehnung an eine ethnografische Vorgehensweise entwickelt und durchgeführt wurde (S. 101). Eine befragte und beobachtete Kursleitergruppe sowie eine teilnehmende Beobachtung eines Bildungsurlaubs bilden neben leitfadengestützten Interviews mit Teilnehmenden des Bildungsurlaubs die Datengrundlage (siehe Kap. 5). Als Auswertungsmethode wählt Schepers die Grounded Theory nach Strauss/Corbin. Die Auseinandersetzung mit den verschiedenen Datensätzen und der Auswertungsmethode erfolgt gründlich, das konkrete Vorgehen mit der Grounded Theory fehlt jedoch.

Im abschließenden Kapitel werden die relevanten Ergebnisse der Untersuchung verdichtet und lokalisierte Forschungsdesiderate gekennzeichnet. Es wurden drei Hauptachsen ausgearbeitet: „Lernwiderstände“, „Berufliches Selbstverständnis“ und „Teilnehmerorientierung“. Kursleiterspezifische Lernwiderstände sind laut Schepers auf die berufliche Rolle von Kursleitenden zurückzuführen und zeigten sich häufig in der Interaktion der teilnehmenden Kursleitenden und der Seminarleitung.

Im Ausblick gibt Schepers mögliche Vorschläge, wie die Professionalisierung des Berufsfelds der Kursleitenden bereichert werden könne. Herausforderungen lägen hier u. a. bei den Rahmenbedingungen des Lernens und einer individuellen Professionalitätsentwicklung.

Den Gesamtkontext der Professionalisierung der Erwachsenen-/Weiterbildung berücksichtigend, ist das Nebenfazit der Autorin interessant. Sie bemerkt, dass Strukturen und Rahmenbedingungen im Bereich der Kursleiterfortbildungen weit weg von einer erwachsenenpädagogischen Professionalisierung sind. Weder das Wissen, das in der untersuchten Fortbildung vermittelt wurde, entstamme der Erwachsenenbildungswissenschaft, noch erfüllten die Rahmenbedingungen des Lernens durchgängig Kriterien, die im Sinne der Erwachsenenbildung wissenschaftlich fundiert wären. Kursleitende sollen also ihre Professionalität für das erwachsenenpädagogische Berufshandeln in einem Rahmen erlernen, der nicht diesen Ansprüchen gerecht wird. Es ließe sich demnach weiterer Forschungsbedarf hinsichtlich der Qualität der Fortbildungsangebote für Kursleitende ableiten. Ein kleiner Wehrmutstropfen hinsichtlich der vorliegenden Forschungsarbeit lässt sich daraus ableiten, denn die Auswahlkriterien für die analysierte Fortbildung werden dem Leser über die Tatsache hinaus, dass es sich dabei um eine kostenfreie Bildungsurlaubsmaßnahme zum Thema „Menschen und ihr Verhalten in der Gruppe“ (S. 103) handelt, nicht transparent gemacht. 
Insgesamt ist es eine gelungene Untersuchung zu einem immer aktuellen Thema. Die empirische Qualifikationsarbeit ist klar gegliedert und bietet unterschiedlichen Lesertypen mit den gewählten Überschriften eine leserfreundliche Struktur.

Open Access Dieser Artikel wird unter der Creative Commons Namensnennung 4.0 International Lizenz (http://creativecommons.org/licenses/by/4.0/deed.de) veröffentlicht, welche die uneingeschränkte Nutzung, Verbreitung und Wiedergabe für beliebige Zwecke erlaubt, sofern Sie den/die ursprünglichen Autor(en) und die Quelle ordnungsgemäß nennen, einen Link zur Creative Commons Lizenz beifügen und angeben, ob Änderungen vorgenommen wurden. 